\title{
Social mobilization and social marketing to promote NaFeEDTA-fortified soya sauce in an iron-deficient population through a public-private partnership
}

\author{
Bo Wang ${ }^{1}$, Siyan Zhan ${ }^{1}$, Jing Sun ${ }^{2}$ and Liming Lee ${ }^{1, *}$ \\ 'Department of Epidemiology and Biostatistics, School of Public Health, Peking University Health Science Center, \\ 38 Xueyuan Road, Haidian District, Beijing 100083, People's Republic of China: ${ }^{2}$ The Institute of Nutrition and \\ Food Safety, Chinese Center for Disease Control and Prevention, 29 Nan Wei Road, Beijing 100050, People's \\ Republic of China
}

Submitted 16 June 2008: Accepted 28 0ctober 2008: First published online 23 December 2008

\begin{abstract}
Objective: The present pilot project aimed to assess the effectiveness of social mobilization and social marketing in improving knowledge, attitudes and practices (KAP) and Fe status in an Fe-deficient population.

Design: In an uncontrolled, before-after, community-based study, social mobilization and social marketing strategies were applied. The main outcomes included $\mathrm{KAP}$ and $\mathrm{Hb}$ level and were measured at baseline, 1 year later and 2 years later. Setting: One urban county and two rural counties in Shijiazhuang Municipality, Hebei Province, China.

Subjects: Adult women older than 20 years of age and young children aged from 3 to 7 years were selected from three counties to attend the evaluation protocol. Results: After 1 year, most knowledge and attitudes had changed positively towards the prevention and control of anaemia. The percentage of women who had adopted NaFeEDTA-fortified soya sauce increased from 8.9\% to 36.6\% $(P \leq 0 \cdot 001)$. After 2 years, Hb levels had increased substantially, by $9 \cdot 0 \mathrm{~g} / 1$ $(P \leq 0 \cdot 001)$ in adult women and $7 \cdot 7 \mathrm{~g} / 1(P \leq 0 \cdot 001)$ in young children.

Conclusion: Social mobilization and social marketing activities had a positive impact on the KAP of adult women, and resulted in marked improvements in $\mathrm{Hb}$ levels in both adult women and young children. This should be recommended as a national preventive strategy to prevent and control Fe deficiency and Fedeficiency anaemia.
\end{abstract}

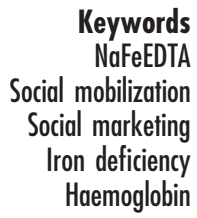

Keywords

MFeEDTA

Social marketing Haemoglobin
Fe deficiency is a major health problem in developing countries and affects about 3.5 billion people throughout the world, mainly women of reproductive age, infants and young children ${ }^{(1)}$. Fe-deficiency anaemia (IDA) is the most severe form of Fe deficiency. According to a WHO report $^{(2)}$, in 2001 over 2 billion people suffered from IDA. In China, the National Nutrition and Health Survey in 2002 reported a high prevalence of anaemia: about $10 \%$ in young children and $20 \%$ in adult women ${ }^{(3)}$. Low Fe bioavailability, which is mainly due to the presence of inhibitors such as phytic acid from cereal-based diets, has been identified as the major cause of IDA in China ${ }^{(3-6)}$. The impact of IDA on health manifests in the following four aspects ${ }^{(7-12)}$ : (i) IDA leads to low birth weight, increased maternal and neonatal mortality, and increased infant mortality; (ii) in infancy, IDA delays physical and mental development and thus damages work capacity in adulthood; (iii) in children, IDA increases the chances and prolongs the duration of upper respiratory tract infections; and (iv) as anaemia damages capacity related to oxygen transport and lowers tolerance, the physical strength and work capacity of all IDA individuals is harmed and this undoubtedly leads to decreased income on an individual, family and country level.

The fortification of foods is often regarded as the most cost-effective long-term approach to reducing the prevalence of Fe deficiency ${ }^{(13,14)}$. Compared with the commonly used Fe salt fortificants, NaFeEDTA is characterized by a higher absorption rate in the human body, fewer adverse effects and less influence on the bioavailability of other minerals ${ }^{(14,15)}$. A therapeutic trial conducted in anaemic schoolchildren in China found that the daily administration of $5 \mathrm{mg}$ of Fe from NaFeEDTA in $5 \mathrm{ml}$ of soya sauce cured all cases of anaemia in 3 months ${ }^{(16)}$. The effectiveness of NaFeEDTA-fortified soya sauce for controlling Fe deficiency was demonstrated in an 18-month, randomized, placebo-controlled intervention trial in a highrisk population ${ }^{(17)}$. Although the efficacy and effectiveness 
of NaFeEDTA-fortified soya sauce have been demonstrated under strictly supervised programme conditions by previous studies, the effectiveness of any promotion strategy also needs to be demonstrated before policy decisions are taken to launch a national preventive strategy.

In recent years, social mobilization and social marketing have been suggested as an effective way to achieve behaviour change in health and nutrition promotion programmes $^{(18)}$. Therefore, the specific objectives of the present pilot project were to promote the adoption of NaFeEDTA-fortified soya sauce among an Fe-deficient population through social mobilization and social marketing and to assess the effectiveness of such an approach in improving the knowledge, attitudes and practices (KAP) and Fe status of the Fe-deficient population in the control of the IDA.

\section{Subjects and methods}

\section{Study design and subjects}

In early 2004, the Food Fortification Office (FFO) of the Chinese Center for Disease Control and Prevention (CDC) designed the study protocol, which was approved by the institutional review board of the Institute of Nutrition and Food Safety (INFS) at the Chinese CDC. The study was an uncontrolled, community-based, before-after study, which was implemented for 2 years between October 2004 and October 2006 in three selected counties (one urban county, Chang'an, and two rural counties, Luquan and Zanhuang) of Shijiazhuang Municipality, capital of Hebei Province, located $280 \mathrm{~km}$ to the south of Beijing. These three counties are composed of thirty-five rural communes/townships and 550 rural villages/urban communities, with a population of nearly 1 million and an area of about $1923 \mathrm{~km}^{2}$.

A strong social mobilization and social marketing effort accompanied the programme. The main outcomes included KAP and Hb level. Adult women (non-pregnant) older than 20 years of age and children aged 3 to 7 years were selected to participate in the evaluation protocol considering the following reasons: (i) adult women and pre-school children are high-risk populations according to the 2002 National Survey ${ }^{(3)}$; (ii) in Chinese families, adult women are the main purchasing population for soya sauce; and (iii) some schools provide meals to students and are beyond the original scope of our study, which was designed to address the effect of intervention on individual behaviour. Thus, school-aged children were excluded from the evaluation.

Cross-sectional samples were used to assess different groups of individuals in each survey. Because this was a pilot study and no data existed predicting the baseline KAP and Fe status of the population, we did not calculate a sample size or use power calculations in the design phase of the study. Stratified quota sampling was used in the sampling procedure. For biological monitoring, the urban county and one of the two rural counties were involved and about 1000 subjects (800 adult non-pregnant women older than 20 years of age and 200 children aged 3 to 7 years) were sampled at each survey: 200 adult women from one community of the urban county, and 600 adult women and 200 young children from two villages of the rural county (300 adult women and 100 young children from each village) were selected. The proportion of the number of adult women from each age period (20-29, 30-39, 40-49, 50-59, older than 60 years) in each selected community (village) was 1:1:1:1:1. For KAP investigation, all three counties were involved at each survey: 200 adult women from two communities of the urban county (100 from each community) and 200 adult women from four villages of the two rural counties (two villages from each county and fifty women from each village) were selected. Among the three surveys, different communities (villages) were sampled.

\section{Key intervention strategies}

The project was implemented by FFO in collaboration with Zhenji Brew Group Company of China, the largest condiment enterprise (Joint Stock Company) in Hebei Province. Thus the project team included national coordinators, nutritionists and epidemiologists, marketing consultants, a representative from the enterprise, and programme management personnel. The project team, in close coordination with the health administration departments at the levels of province, municipality and county, conducted the following social mobilization and social marketing activities.

\section{Social mobilization}

Social mobilization is a broad-scale movement to engage people's participation and create a supportive environment in achieving a specific development goal. In the present project it comprised the following steps.

1. The project began with a kick-off meeting. The project team, representatives from the Ministry of Health, local health administration departments and CDC, and heads of local government attended this meeting. Officials and organizations in the project area were mobilized to obtain their leadership support and to ensure the participation of all sectors.

2. Village/community health workers, village/community heads and store owners/market managers received training to increase their knowledge about Fe deficiency and IDA, orient them about the project, identify their potential roles in project implementation, improve their skills in counselling, and strengthen skills in marketing (for store owners and supermarket managers).

3. Schoolchildren were mobilized to distribute information, education and communication (IEC) materials to the whole family after they learned the relevant knowledge through specially designed classes. 
4. Trained volunteers were recruited to disseminate IEC materials in public areas like hospitals, culture and sports centres.

\section{Social marketing}

Social marketing, which is the design, implementation and control of programmes seeking to increase the acceptability of a social idea or behaviour, was used to promote the NaFeEDTA-fortified soya sauce. After formative research, the following strategies in the present project were conducted jointly by FFO and the Zhenji Company.

Product. NaFeEDTA has a high Fe bioavailability in the human body through protection against inhibition by phytic acid ${ }^{(19)}$. Experiments have shown that the bioavailability of Fe in NaFeEDTA is two to three times higher than the traditional $\mathrm{Fe}$ preparation, $\mathrm{FeSO}_{4}$, which is generally regarded as having a relatively high bioavailability of Fe compared with other Fe preparations ${ }^{(19,20)}$. Soya sauce is a commonly used condiment in China and NaFeEDTA does not cause organoleptic changes in soya sauce ${ }^{(17)}$. Thus it was selected as the food carrier for NaFeEDTA. Production techniques of food-grade NaFeEDTA and NaFeEDTA-fortified soya sauce were developed by the INFS, Chinese CDC. Efficacy and effectiveness studies implemented by the INFS have demonstrated that NaFeEDTA-fortified soya sauce is highly effective in controlling Fe deficiency and reducing the prevalence of $\operatorname{IDA}^{(16,17)}$. Under the promotion of INFS, the Ministry of Health of China approved NaFeEDTA as a nutrient fortificant for soya sauce based on evidence provided by previous studies. The approved concentration is $175-210 \mathrm{mg} / 100 \mathrm{ml}$ soya sauce $(23-27 \mathrm{mg}$ $\mathrm{Fe} / 100 \mathrm{ml}$ ). In our project, NaFeEDTA-fortified soya sauce was produced by the Zhenji Company. The benefits of this product were defined as 'the desire to keep the family healthy and energetic', 'the desire to make women charming' and 'the desire to make children more intelligent'. A special logo was designed and used on all NaFeEDTA-fortified soya sauce and promotional materials to brand this product. The logo included a red blob with a Chinese word 'iron' in the middle of this blob, which indicated the importance of Fe to health. The names Chinese CDC and China Flavoring Industry were designed to appear near the blob to indicate the authority responsible for ensuring that the products were safe and effective.

Price. In the project, NaFeEDTA-fortified soya sauce products were merely made available in the market, rather than being supplied free of charge. The selling price was a little higher than unfortified soya sauce so that it was not under-priced compared with production costs. However, the estimated annual additional cost of NaFeEDTA-fortified soya sauce would be less than \$US 0.5 per person and suitable to the economic condition of the intervention population. In addition to monetary costs, there were concerns about safety, taste and appearance. The target audience was made aware about the safety issue through being informed of approval from the Government and a guarantee from Chinese CDC. In market and promotion activities, taste tests were designed to overcome the perception that NaFeEDTA-fortified soya sauce did not appear or taste as good as unfortified soya sauce.

Place. In the project area, the Zhenji Company has established highly matured transportation, distribution and sales networks in collaboration with supermarkets and retail stores for many years. Through the existing network, NaFeEDTA-fortified soya sauce products were supplied for sale. They were placed in prominent locations in supermarkets and retail stores to provide easy and convenient access for consumers. In addition to markets, popular neighbourhood locations (e.g. public activity spaces, hospitals, culture and sports centres) were focused on for IEC and promotion. The Place factors of the project strengthened receptivity for both the information and the product.

Promotion. IEC materials were developed, produced, distributed and disseminated to the intervention population. These materials were in the form of leaflets, foldouts, billboards, banners, wall slogans, newsletters, short videos and public service announcements. The key contents of these IEC materials were the importance of Fe to health and how to prevent Fe deficiency and IDA through using NaFeEDTA-fortified soya sauce. During each campaign, leaflets and foldouts were distributed and used to communicate messages. Billboards were placed in schools, markets, health clinics and bus stations, and banners were suspended in strategic places during the campaign periods. Slogans were painted on the walls in rural villages. Newsletters were released in newspapers, distributed in markets and disseminated by volunteers. Public service announcements, which were followed closely by commercial advertisements for products from the Zhenji Company, were broadcast on television and radio. Shopping bags with product benefit were provided and put into use in markets. Those retail stores and supermarkets where NaFeEDTA-fortified soya sauce was on sale were awarded with a special shop sign by the local government. Point-of-decision prompts were used by trained salespersons to encourage and persuade consumers to purchase fortified soya sauce. Signature campaigns, held in public activity spaces, were characterized by participation of local government leaders, provision of nutritionists' counselling and taste tests, performance of tradition culture, and a free bottle of fortified soya sauce per person. Educational videos were shown before specially offered movies started in rural villages, where outside movies are very popular. All these campaigns were aimed at informing the target populations about project activities and promoting the participation of all those involved in the prevention and control of IDA. 


\section{Data collection and analysis}

Three surveys were conducted, one each year: at baseline, and then 1 and 2 years later to evaluate the effect of the intervention on KAP and $\mathrm{Hb}$ level. At each survey blood samples were collected and analysed for $\mathrm{Hb}$. KAP were measured only at two surveys, baseline and 1 year later through a questionnaire developed by FFO. Following WHO criteria, anaemia in adult non-pregnant women was defined as $\mathrm{Hb} \leq 120 \mathrm{~g} / \mathrm{l}$ and in children aged 3 to 7 years as $\mathrm{Hb} \leq 110 \mathrm{~g} / \mathrm{l}^{(21)}$. The Zhenji Company sales records were monitored over the project period as one indicator of adoption. When appropriate, data were analysed using the Statistical Package for the Social Sciences statistical software package version $13 \cdot 0$ (SPSS Inc., Chicago, IL, USA). ANOVA and post hoc multiple comparisons (Least Significant Difference $t$ test) were applied for $\mathrm{Hb}$ levels. The $\chi^{2}$ test was used for anaemia prevalence comparisons among the three surveys and KAP comparisons between the two surveys. A Bonferroni correction was used to allow for multiple comparisons of anaemia prevalence data among the three surveys.

\section{Results}

\section{Impact on knowledge, attitudes and practices of adult women}

Results from the KAP surveys are shown in Table 1. As social marketing and social mobilization activities were implemented, KAP changes occurred over the 12 months of the intervention. Only $46 \cdot 1 \%$ of the women initially were aware that Fe deficiency led to anaemia; however, awareness increased to over $85 \%$ after 1 year $(P \leq 0 \cdot 001)$. Notable changes were also observed in awareness of the symptoms of Fe deficiency, the impacts of Fe deficiency on children and the population susceptible to Fe deficiency: with time, levels of most knowledge increased remarkably, and the percentages of adult women with 'have no idea' or 'no impact (on children)' decreased significantly. However, the percentages of women who knew that a pale face was one symptom that resulted from Fe deficiency, and that young children and the old were susceptible to Fe deficiency, unexpectedly decreased. In terms of attitude on measures undertaken to control Fe deficiency, there was also a marked increase in the percentage of adult women who would improve their diet (including taking Fe-fortified food), which almost doubled $(P \leq 0 \cdot 001)$, and the percentage of women who would not take any measures decreased from $6 \cdot 6 \%$ to $1 \cdot 3 \%$ $(P \leq 0 \cdot 001)$. All the promotion activities in the project were geared towards promoting and encouraging the behaviour of adopting NaFeEDTA-fortified soya sauce. The KAP survey showed that the percentage of women who had ever bought the fortified soya sauce increased markedly from $8.9 \%$ to $36 \cdot 6 \%(P \leq 0 \cdot 001)$.

As shown in Fig. 1, the half-year sales of NaFeEDTAfortified soya sauce grew impressively through the project period, increasing from 188 tons before implementation to 1480 tons at the end of the project. This was an increase of almost seven-fold over the baseline of the project.

Table 1 Changes in knowledge, attitudes and practices (KAP) of adult non-pregnant women, aged 20 years or older, about iron and anaemia between baseline (survey 1) and 1 year later (survey 2) following social mobilization and social marketing activities, Hebei Province, China, October 2004-October 2005

\begin{tabular}{|c|c|c|c|}
\hline \multirow[b]{2}{*}{ KAP } & \multicolumn{2}{|c|}{ Survey (\%) } & \multirow[b]{2}{*}{$P$} \\
\hline & 1 & 2 & \\
\hline I know Fe deficiency leads to anaemia & $46 \cdot 1$ & $85 \cdot 5$ & $\leq 0 \cdot 001$ \\
\hline \multicolumn{4}{|l|}{ Fe deficiency results in the following symptoms } \\
\hline A pale face & $21 \cdot 4$ & $13 \cdot 7$ & 0.004 \\
\hline Infirm & $17 \cdot 2$ & $21 \cdot 1$ & $\leq 0.05$ \\
\hline Fatigable & $16 \cdot 0$ & $40 \cdot 9$ & $\leq 0.001$ \\
\hline Intolerance to cold & $3 \cdot 1$ & $40 \cdot 9$ & $\leq 0.001$ \\
\hline Easy to catch a cold and low resistance & $10 \cdot 1$ & $32 \cdot 7$ & $\leq 0.001$ \\
\hline Have no idea & $54 \cdot 6$ & $16 \cdot 4$ & $\leq 0 \cdot 001$ \\
\hline \multicolumn{4}{|l|}{ Impacts of Fe deficiency on children } \\
\hline Weak body conditions & $15 \cdot 5$ & $12 \cdot 4$ & $\leq 0.05$ \\
\hline Impaired mental development & $18 \cdot 1$ & $34 \cdot 8$ & $\leq 0.001$ \\
\hline Delayed physical development & $24 \cdot 2$ & $40 \cdot 6$ & $\leq 0.001$ \\
\hline Have no idea & $56 \cdot 0$ & $41 \cdot 2$ & $\leq 0.001$ \\
\hline No impact & $5 \cdot 2$ & 0 & $\leq 0.001$ \\
\hline \multicolumn{4}{|l|}{ Who are susceptible to Fe deficiency } \\
\hline Infants & $7 \cdot 8$ & $5 \cdot 8$ & $\leq 0.05$ \\
\hline Young children & $39 \cdot 8$ & $31 \cdot 7$ & 0.015 \\
\hline Adolescents & $1 \cdot 9$ & $44 \cdot 3$ & $\leq 0.001$ \\
\hline Women & $23 \cdot 5$ & $37 \cdot 2$ & $\leq 0.001$ \\
\hline The old & $25 \cdot 9$ & $10 \cdot 8$ & $\leq 0.001$ \\
\hline Have no idea & $44 \cdot 0$ & $10 \cdot 0$ & $\leq 0.001$ \\
\hline \multicolumn{4}{|c|}{ If Fe deficiency is identified, what measures will be undertaken } \\
\hline No measures & $6 \cdot 6$ & $1 \cdot 3$ & $\leq 0.001$ \\
\hline Improve diet (including taking Fe-fortified food) & $41 \cdot 2$ & $82 \cdot 1$ & $\leq 0.001$ \\
\hline I have ever bought Fe-fortified soya sauce & 8.9 & $36 \cdot 6$ & $\leq 0.001$ \\
\hline
\end{tabular}




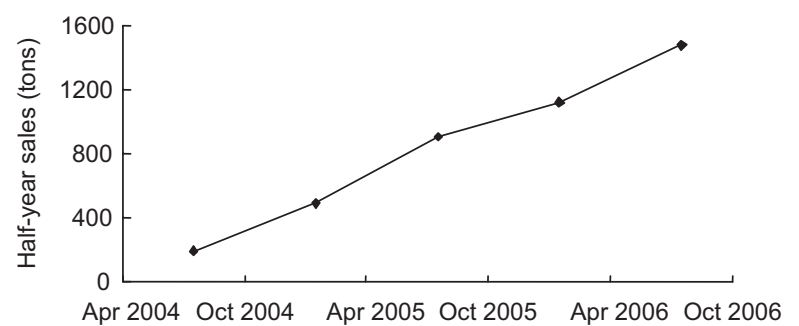

Fig. 1 Half-year sales of NaFeEDTA-fortified soya sauce (source: Zhenji Company, Hebei, China)

\section{Impact on anaemia in adult women and young children}

Table 2 presents the changes in $\mathrm{Hb}$ values at the three surveys for the adult women and young children. In both adult women and young children, significant differences were found among the three surveys $(P \leq 0 \cdot 001)$, with $\mathrm{Hb}$ concentrations increasing significantly from survey 1 to survey 2 , and again from survey 2 to survey 3 . Figures 2 and 3 show that, from survey 2 on, $\mathrm{Hb}$ distributions were shifted to higher concentrations compared with those at survey 1 , and the tail of low $\mathrm{Hb}$ was almost eliminated at survey 3 .

In adult women, anaemia prevalence was significantly different at the three surveys ( $P \leq 0 \cdot 001$, Table 2$)$. Multiple comparisons revealed that anaemia decreased from baseline to survey 2 and again from survey 2 to survey 3 . However, in young children, no difference in anaemia prevalence existed among the three surveys $(P=0 \cdot 12$, Table 2).

\section{Discussion}

\section{Study design and intervention strategies}

In many community-based intervention studies, assessing all individuals of interest may not be feasible. In the current project, a cross-sectional sampling approach, rather than cohort samples, was applied. Although longitudinal cohort analyses will typically have greater statistical power than repeated cross-sectional analyses ${ }^{(22-25)}$, repetitive assessments may increase participants' knowledge of healthy behaviour and artificially influence behaviour, which can confound results ${ }^{(26)}$. Thus, it has been argued that repeated cross-sectional analyses are more appropriate than cohort analyses for measuring the effectiveness of interventions in the community ${ }^{(22,27,28)}$.

The absence of a placebo group, owing to ethical reasons, was a limitation of the present study. Secular trends in KAP, Hb level and anaemia prevalence may confound the interpretation of this before-after study. However, this possibility was not supported by available evidence. First, the relatively stable socio-economic status (SES) in the intervention area over the 2-year project period and the lack of any other interventions to control
Table $2 \mathrm{Hb}$ and anaemia prevalence at each of three surveys in adult non-pregnant women aged 20 years or older and young children aged 3 to 7 years, Hebei Province, China, October 2004October 2006

\begin{tabular}{lcccc}
\hline & \multicolumn{3}{c}{ Survey $^{*}$} & \\
\cline { 2 - 4 } & 1 & 2 & 3 & $P+$ \\
\hline Adult women & & & & \\
$\quad$ No. of subjects & 801 & 716 & 787 & \\
Hb (g/l) & & & & \\
$\quad$ Mean & $119 \cdot 4^{\mathrm{a}}$ & $124 \cdot 4^{\mathrm{b}}$ & $128 \cdot 4^{\mathrm{c}}$ & $\leq 0.001$ \\
$\quad$ SD & $17 \cdot 2$ & $15 \cdot 1$ & $11 \cdot 2$ & \\
Anaemia (\%) & $42 \cdot 3^{\mathrm{a}}$ & $31 \cdot 4^{\mathrm{b}}$ & $22 \cdot 9^{\mathrm{c}}$ & $\leq 0.001$ \\
Young children & & & & \\
$\quad$ No. of subjects & 198 & 200 & 175 & \\
Hb (g/l) & & & & \\
$\quad$ Mean & $118 \cdot 8^{\mathrm{a}}$ & $122 \cdot 6^{\mathrm{b}}$ & $126 \cdot 5^{\mathrm{c}}$ & $\leq 0 \cdot 001$ \\
$\quad$ SD & $12 \cdot 5$ & $10 \cdot 5$ & $9 \cdot 7$ & \\
Anaemia (\%) & $17 \cdot 2$ & $12 \cdot 0$ & $10 \cdot 3$ & 0.12 \\
\hline
\end{tabular}

$\overline{a, b, c}$ Mean values within a row with unlike superscript letters were significantly different between surveys.

*Survey 1 was at baseline; survey 2 and survey 3 were respectively 1 year and 2 years later, following social mobilization and social marketing activities.

tANOVA and post hoc Least Significant Difference $t$ test for $\mathrm{Hb} ; \chi^{2}$ test and Bonferroni correction for anaemia prevalence.

anaemia did not suggest a significant natural change. Second, a survey carried out in Handan City (geographically adjacent to and very similar in SES and dietary habits to our project area) of Hebei Province, in the middle of 2005 , revealed that the anaemia prevalence was $40.5 \%$ in adult women and $17 \cdot 5 \%$ in children aged 3 to 7 years $^{(29)}$. This result was very similar to the baseline of our project and indicated that secular trends seemed not to be in play. Third, sales of the NaFeEDTA-fortified soya sauce were included in the project as an outcome. The rapid increase in this outcome, to some extent, confirmed the link between the social marketing and social mobilization intervention and the final $\mathrm{Hb}$ level and anaemia prevalence outcomes.

Social marketing is one approach which draws from successful techniques used by commercial marketers. Since the 1960s, it has been applied to promote traffic safety, tobacco control, drug prevention, childhood immunizations and environmental behaviour, as well as improved nutrition and diets, with significant success ${ }^{(30)}$. According to our knowledge, the present study is the first programme for improving public nutrition using social marketing strategies in China. Strong support from local authorities and active community participation are crucial for community-based health and nutrition programmes to be successful. Social mobilization approach has been effectively used by two primary health-care programmes in Vietnam ${ }^{(31)}$. The national expanded programme on immunization and the vitamin A supplementation programme with vitamin A capsules provided twice yearly free of charge by UNICEF to children under the age of 5 , both initiated in the early 1990s, were successful because of the participation of many people and a high 


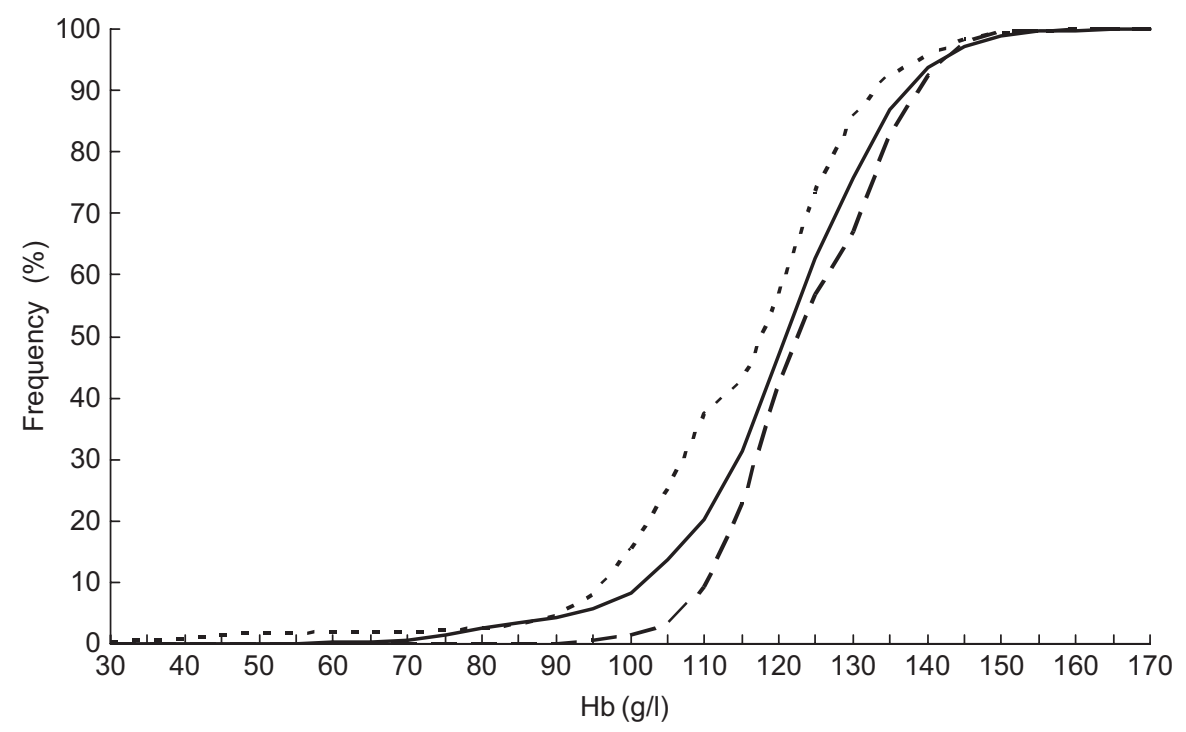

Fig. $2 \mathrm{Hb}$ distributions in adult non-pregnant women, aged 20 years or older, at each of three surveys: baseline (survey 1; - --), and 1 year later (survey $2 ;-$ ) and 2 years later (survey $3 ;---$ ) following social mobilization and social marketing activities, Hebei Province, China, October 2004-October 2006

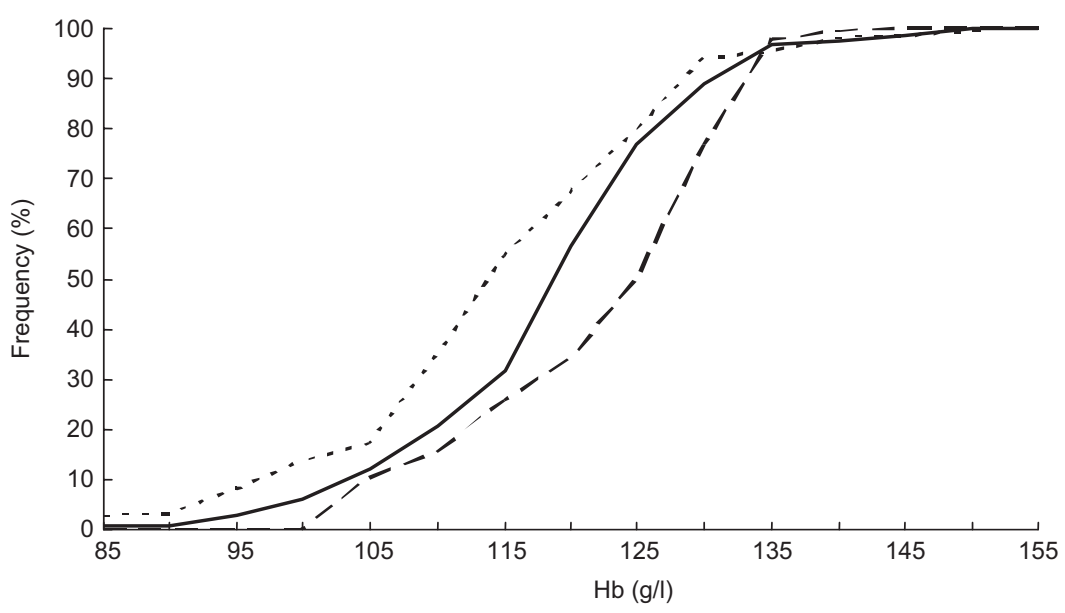

Fig. $3 \mathrm{Hb}$ distributions in young children aged 3 to 7 years at each of three surveys: baseline (survey 1 ; - - -), and 1 year later (survey $2 ;-$ ) and 2 years later (survey $3 ;---$ ) following social mobilization and social marketing activities, Hebei Province, China, October 2004-October 2006

commitment by authorities from different levels of government and the community. Besides, social mobilization had helped construct part of the distribution system for Fe-folic acid supplements by mobilizing the Women's Union network to overcome the shortcomings of an undeveloped market system in another project in Vietnam $^{(32)}$. In our project, formative research had suggested that winning the trust of the public on NaFeEDTA-fortified soya sauce was a major barrier, as plenty of advertisements for many kinds of functional foods appeared frequently in mass media. Thus one important role of social mobilization in the present project was to show government support for this product.

\section{Study results}

Overall, the intervention strategies contributed significantly to changing the KAP of adult women. However, levels of three knowledge items decreased. It was possible that the complexity of the KAP questionnaire had caused these unexpected findings. In our questionnaire, most questions on knowledge had multiple options. This made the question answering process more difficult compared with those questionnaires consisting of 'I agree/I disagree' statements. Undoubtedly, these findings indicate that such knowledge bases need to be strengthened by future promotional activities and IEC materials. Anaemia was a public health problem in $42 \cdot 3 \%$ of adult 
women in the present study. This prevalence was much higher than the national prevalence of anaemia in adult women of the 2002 National Nutrition and Health Survey ${ }^{(3)}$. After 2 years of intervention, the prevalence decreased dramatically to $22.9 \%$. The $\mathrm{Hb}$ levels of the adult women increased substantially $(P \leq 0 \cdot 001)$ and the increment, $9 \cdot 0 \mathrm{~g} / \mathrm{l}$, was substantial and of great significance to public health. In children, the intervention achieved an increment of $7 \cdot 7 \mathrm{~g} / 1$ in $\mathrm{Hb}$ levels after 2 years $(P \leq 0 \cdot 001)$. Although the decline in prevalence $(6.9 \%)$ was not statistically significant $(P=0 \cdot 12)$, it was substantial and practically important. It was possible that the data may have been inadequate to detect an association due to the relatively small number of subjects.

Soya sauce is an important component of the Chinese diet; more than $70 \%$ of households consume soya sauce in two or three meals a day. The average consumption is estimated to be $16.4 \mathrm{ml}$ per person per day ${ }^{(17)}$. As the concentration of Fe in NaFeEDTA-fortified soya sauce is about $25 \mathrm{mg} \mathrm{Fe} / 100 \mathrm{ml}$, the expected intake of Fe from the fortified soya sauce in our project would be $4.1 \mathrm{mg}$ per person per day.

At the end of the study, the prevalence of anaemia was still high in both adult women and young children. The following possible explanations have been considered. First, the 2-year duration of the intervention may have not been long enough to achieve lower prevalence. Biological changes caused by NaFeEDTA would not appear until fortified soya sauce was voluntarily adopted. Second, due to considerations of convenience and cost, anaemia rather than IDA was employed as one outcome in this project. Although Fe deficiency is the leading cause of anaemia worldwide ${ }^{(33)}$, it is not the only reason for anaemia, which can also result from other micronutrient deficiencies, such as folic acid, vitamin $\mathrm{B}_{12}$, vitamin $\mathrm{A}$ or riboflavin ${ }^{(32,34,35)}$. Thus it is possible that other nutritional deficiencies have contributed to the final prevalence of anaemia.

Only adult women and young children were measured in the present study; however, it was reasonable to deduce that other populations had probably also benefited in KAP and Fe status from the project. In the project, promotional activities were designed and implemented for all the population. Fortified soya sauce, once adopted, would be consumed by the whole family.

Similar to our project, the social marketing and social mobilization strategy has also been effective in several programmes aimed to promote Fe-folic acid supplementation in women of reproductive age. In the programme launched in Vietnam ${ }^{(31,32)}$, the approach created a significant positive impact on changing KAP of reproductive-aged women and the anaemia prevalence in non-pregnant women in this group decreased from $45 \cdot 6 \%$ to $19 \cdot 1 \%(P \leq 0 \cdot 05)$ after 1 year. The Cambodian programme was implemented in three different environments (secondary school girls, women working in garment factories and women in rural villages) and found that all three groups of reproductive-aged women showed substantial improvements in knowledge about the causes, consequences and prevention of anaemia $^{(36)}$. In the Philippine programme ${ }^{(37-39)}$, knowledge and practice of buying and regularly taking supplements by reproductive-aged women were both improved. Although $\mathrm{Hb}$ levels showed minimal change, serum ferritin and haematocrit increased significantly.

We believe that the present project will probably have good sustainability. The Zhenji Company currently has the capacity to produce and stably supply NaFeEDTAfortified soya sauce locally for use. Well-developed transportation, distribution and sales networks provided convenient access to the public. Unlike other programmes in which supplements were provided free of charge and under supervised conditions, NaFeEDTA-fortified soya sauce was sold in our project. The public, therefore, voluntarily chose to purchase the product since they had to pay for it. However, the price was affordable even to those with low economic status. Thus, the price strategies had assured both the for-profit purpose of industry and acceptability to the public.

The social marketing and mobilization activities emphasized the '5Ps' approach: public relations and collaboration, product, price, place and promotion ${ }^{(31)}$. For such an approach to be successful, a strong public-industry partnership is needed ${ }^{(40)}$. It has been recognized that for many large-scale health-related challenges, conventional approaches to programme development traditionally carried out by the public sector can yield only limited results ${ }^{(41)}$. Such partnerships can formalize the sharing of expertise and resources between the public and private sectors for the purpose of a specific healthcare challenge ${ }^{(42)}$, and make both sectors derive significant benefit ${ }^{(40)}$. In our project, the public sector and industry successfully worked together to develop and implement strategies: the institution (INFS) affiliated with the public sector (Chinese CDC) developed the production techniques of NaFeEDTA-fortified soya sauce and assessed the efficacy and effectiveness in controlling Fe deficiency and anaemia; the Ministry of Health approved its use in the market; the industry (the Zhenji Company) produced and marketed NaFeEDTA-fortified soya sauce that was reasonably priced; the public sector (FFO, local government, local health administration and CDC) assumed a leading role in building public relations, collaboration and mobilization; the public sector and the industry sector co-operated to design consistent educational messages and conducted promotional activities, which were characterized by government support from the public sector and financial support from the industry.

\section{Conclusion}

The findings of the present pilot project demonstrate the effectiveness of social mobilization and social marketing 
strategies, through a public-private partnership, in creating a significantly positive impact on changing the knowledge and attitudes of adult women about Fe deficiency and anaemia, promoting the purchasing of NaFeEDTA-fortified soya sauce, and improving Fe status, as expressed by $\mathrm{Hb}$ levels, of adult women and young children. The project also proved that such social mobilization and social marketing strategies are feasible in a Chinese context and could be adopted as a national preventive programme in the prevention and control of Fe deficiency and IDA.

\section{Acknowledgements}

The Global Alliance for Improved Nutrition (GAIN) funded the study. None of the authors had any conflicts of interest. L.L. and J.S. were responsible for the design and implementation of the project. J.S. was responsible for data management and data collection. B.W. and S.Z. contributed to the development of the study protocol and performed data analysis. B.W., S.Z. and L.L. were responsible for the preparation of the manuscript. All of the authors took part in the discussion of the results and contributed to the drafting of the final version of the manuscript. The authors are grateful to all of the adult women and young children who participated in the evaluation protocol and to the field workers and other personnel from FFO, INFS, the Zhenji Company and health authorities in Hebei Province, Shijiang Municipality and the three selected counties for their collaboration. The technical assistance of Ms Bo Wang during the data collection and statistical evaluation is gratefully acknowledged.

\section{References}

1. United Nations Administrative Committee on Coordination, Sub-Committee on Nutrition (2000) Fourth Report on the World Nutrition Situation: Nutrition Throughout the Life Cycle. Geneva: ACC/SCN in collaboration with the International Food Policy Research Institute.

2. World Health Organization/UNICEF/United Nations University (2001) Iron Deficiency Anaemia Assessment, Prevention and Control: A Guide for Programme Managers. Geneva: WHO.

3. Ministry of Heath of China, Ministry of Science and Technology of China \& National Bureau of Statistics of China (2005) The Nutrition and Health Status of the Chinese People. Beijing: People's Medical Publishing House.

4. Hallberg L, Brune M \& Rossander L (1989) Iron absorption in man: ascorbic acid and dose-dependent inhibition by phytate. Am J Clin Nutr 49, 140-144.

5. Sayers MH, Lynch SR, Charlton RW, Bothwell TH, Walker $\mathrm{RB}$ \& Mayet F (1974) Iron absorption from rice meals cooked with fortified salt containing ferrous sulphate and ascorbic acid. Br J Nutr 31, 367-375.

6. Hurrell RF, Lynch S, Bothwell T et al. (2004) Enhancing the absorption of fortification iron. Int J Vitam Nutr Res $\mathbf{7 4}$, 387-401.
7. Preziosi P, Prual A, Galan P, Daouda H, Boureima H \& Hercberg S (1997) Effect of iron supplementation on the iron status of pregnant women: consequences for newborns. Am J Clin Nutr 66, 1178-1182.

8. Ramakrishnan U (2001) Functional consequences of nutritional anemia during pregnancy and early childhood. In Nutritional Anemias, pp. 43-68 [U Ramakrishna, editor]. Boca Raton, FL: CRC Press.

9. Lozoff B (2000) Perinatal iron deficiency and the developing brain. Pediatr Res 48, 137-139.

10. Angulo-Kinzler RM, Peirano P, Lin E, Garrido M \& Lozoff B (2002) Spontaneous motor activity in human infants with iron-deficiency anemia. Early Hum Dev 66, 67-79.

11. De-Silva A, Atukorala S, Weerasinghe I \& Ahluwahlia N (2003) Iron supplementation improves iron status and reduces morbidity in children with or without upper respiratory tract infections: a randomized controlled study in Colombo, Sri Lanka. Am J Clin Nutr 77, 234-241.

12. Haas JD \& Brownlie TT (2001) Iron deficiency and reduced work capacity: a critical review of the research to determine a causal relationship. J Nutr 131, 676S-688S.

13. Cook JD \& Reusser M (1983) Iron fortification: an update. Am J Clin Nutr 38, 648-659.

14. Hurrell RF (1997) Preventing iron deficiency through food fortification. Nutr Rev 55, 210-222.

15. Hurrell RF, Ribas R \& Davidsson L (1994) Sodium iron EDTA as a food fortificant: influence on zinc, calcium and copper metabolism in the rat. Br J Nutr 71, 85-93.

16. Huo J, Sun J, Miao H et al. (2002) Therapeutic effects of NaFeEDTA-fortified soy sauce in anaemic children in China. Asia Pacific J Clin Nutr 11, 123-127.

17. Chen J, Zhao X, Zhang X et al. (2005) Studies on the effectiveness of NaFeEDTA-fortified soy sauce in controlling iron deficiency: a population-based intervention trial. Food Nutr Bull 26, 177-186.

18. Smitasiri S, Attig GA, Valyasevi A, Dhanamitta S \& Tontisirin K (editors) (1993) Social Marketing Vitamin A-rich Foods in Thailand. A Model Nutrition Communication for Behavior Change Process. Nakhon Pathom: Mahidol University.

19. IVACG Secretariat (1993) A Report of the International Nutritional Anemia Consultative Group: Iron EDTA for Food Fortification. New York: The Nutrition Foundation.

20. Huo JS, Piao JH, Yu B et al. (2003) Study on iron absorption of NaFeEDTA in human body with stable isotope method. Wei Sheng Yan Jiu 32, 19S-24S.

21. World Health Organization/UNICEF/United Nations University (1998) Iron Deficiency Anemia: Prevention, Assessment and Control. Report of a Joint WHO/UNICEF/UNU Consultation. Geneva: WHO.

22. Koepsell TD, Wagner EH, Cheadle AC et al. (1992) Selected methodological issues in evaluating community-based health promotion and disease prevention programs. Annu Rev Public Health 13, 31-57.

23. Farquhar JW, Fortmann SP, Maccoby N et al. (1985) The Stanford Five-City Project: design and methods. Am J Epidemiol 122, 323-334.

24. Diehr P, Martin DC, Koepsell T, Cheadle A, Psaty BM \& Wagner EH (1995) Optimal survey design for community intervention evaluations: cohort or cross-sectional? J Clin Epidemiol 48, 1461-1472.

25. Feldman HA \& McKinlay SM (1994) Cohort versus crosssectional design in large field trials: precision, sample size, and a unifying model. Stat Med 13, 61-78.

26. Atienza AA \& King AC (2002) Community-based health intervention trials: an overview of methodological issues. Epidemiol Rev 24, 72-79.

27. Salonen JT, Kottke TE, Jacobs DR Jr \& Hannan PJ (1986) Analysis of community-based cardiovascular disease prevention studies - evaluation issues in the North Karelia 
Project and the Minnesota Heart Health Program. Int $J$ Epidemiol 15, 176-182.

28. Koepsell TD, Diehr PH, Cheadle A \& Kristal A (1995) Invited commentary: symposium on community intervention trials. Am J Epidemiol 142, 594-599.

29. Food Fortification Office, China Center for Disease Control and Prevention (2005) Results of biological monitoring in Handan City, Hebei Province. http://www.cdc-ffo.cn/ wMcms_ReadNews.asp?NewsID $=693$ (accessed October 2008).

30. Smith WA (2006) Social marketing: an overview of approach and effects. Inj Prev 12, Suppl. 1, i38-i43.

31. Khan NC, Thanh HT, Berger J, Hoa PT, Quang ND, Smitasiri S \& Cavalli-Sforza T (2005) Community mobilization and social marketing to promote weekly iron-folic acid supplementation: a new approach toward controlling anemia among women of reproductive age in Vietnam. Nutr Rev 63, S87-S94.

32. Berger J, Thanh HT, Cavalli-Sforza T, Smitasiri S, Khan NC, Milani S, Hoa PT, Quang ND \& Viteri F (2005) Community mobilization and social marketing to promote weekly iron-folic acid supplementation in women of reproductive age in Vietnam: impact of anemia and iron status. Nutr Rev 63, S95-S108.

33. Fernandez-Ballart J \& Murphy MM (2001) Preventive nutritional supplementation throughout the reproductive life cycle. Public Health Nutr 4, 1363-1366.

34. Ronnenberg AG, Goldman MB, Aitken IW \& Xu X (2000) Anemia and deficiencies of folate and vitamin B-6 are common and vary with season in Chinese women of childbearing age. J Nutr 130, 2703-2710.

35. Son T, Khan N, Sastroamidjojo S \& Wasito E (2003) Effect of multi-vitamin or iron supplementation on hemoglobin, ferritin level and work productivity among Vietnamese anemic female workers. In 8th Asian Food Conference. ASEAN Food Science and Technology: Cooperation and Integration for Development, pp. 244-249 [LD Dien, NK Vu and ND Lam, editors]. Hanoi: Agriculture Publishing House.

36. Kanal K, Busch-Hallen J, Cavalli-Sforza T, Crape B \& Smitasiri S; Cambodian Weekly Iron-Folic Acid Program Team (2005) Weekly iron-folic acid supplements to prevent anemia among Cambodian women in three settings: process and outcomes of social marketing and community mobilization. Nutr Rev 63, S126-S133.

37. Paulino LS, Angeles-Agdeppa I, Etorma UM, Ramos AC \& Cavalli-Sforza T (2005) Weekly iron-folic acid supplementation to improve iron status and prevent pregnancy anemia in Filipino women of reproductive age: the Philippine experience through government and private partnership. Nutr Rev 63, S109-S115.

38. Angeles-Agdeppa I, Paulino LS, Ramos AC, Etorma UM, Cavalli-Sforza T \& Milani S (2005) Government-industry partnership in weekly iron-folic acid supplementation for women of reproductive age in the Philippines: impact on iron status. Nutr Rev 63, S116-S125.

39. Garcia J, Datol-Barrett E \& Dizon M (2005) Industry experience in promoting weekly iron-folic acid supplementation in the Philippines. Nutr Rev 63, S146-S151.

40. Ramakrishnan U \& Yip R (2002) Experiences and challenges in industrialized countries: control of iron deficiency in industrialized countries. I Nutr $\mathbf{1 3 2}$, S820-S824.

41. Widdus R (2001) Public-private partnerships for health: their main targets, their diversity, and their future directions. Bull World Health Organ 79, 713-720.

42. Reich MR (2000) Public-private partnerships for public health. Nat Med 6, 617-620. 